\title{
Comparing the performance of two structural indicators for different water models while seeking for connections between structure and dynamics in the glassy regime
}

\author{
Alejandro R. Verde ${ }^{1}$, Joan Manuel Montes de Oca ${ }^{1}$, Sebastián R. \\ Accordino $^{1}$, Laureano M. Alarcón ${ }^{1}$, and Gustavo A. Appignanesi ${ }^{1 *}$ \\ 1 INQUISUR, Departamento de Química, \\ Universidad Nacional del Sur (UNS)-CONICET, \\ Avenida Alem 1253, 8000 Bahía Blanca, Argentina.
}

(Dated: June 4, 2019)

\begin{abstract}
In this work we compare the performance of two structural indicators based on the degree of translational order up to the second coordination shell in three water models: SPC/E, TIP4P/2005 and TIP5P. Beyond directly contrasting their distributions for different temperatures to evidence their usefulness in estimating the fraction of structured and unstructured molecules and, when possible, their classification capability, we also correlate them with an indirect measure of structural constraint: the dynamic propensity. Furthermore, this procedure enables us to show the existence of evident correlations between structural and dynamical information. More specifically, we find that locally structured molecules display a preference for low dynamic propensity values and, more conspicuously, that locally unstructured molecules are extremely subject to high dynamic propensity. This result is particularly relevant for the supercooled regime where the establishment of firm links between structure and dynamics has remained rather elusive, since the occurrence of dynamics that vary in orders of magnitude upon supercooling usually contrast with barely noticeable overall structural changes.
\end{abstract}

\section{INTRODUCTION}

The comprehension of the structural and dynamical behavior of water is not only a matter of great intrinsic interest but it also becomes essential for several central fields of science and technology [1-14]. But contrary to what its simple molecular structure might suggest, water represents in fact a quite complex system, with several anomalies that get more pronounced as the liquid is cooled down [15-32]. Such anomalies are expected to stem from the existence of two competing preferential local molecular structures: well structured low local density molecules and unstructured high local density ones [15, 16, 18, 21-32] (as will be analyzed in detail later on, by well structured water molecules we mean molecules with a well-developed first neighbors' tetrahedron, separated by a clear gap from the second neighbors' shell). As the liquid is cooled down below the melting temperature (fast enough to avoid crystallization, thus entering in the so-called supercooled regime) the low local density state is expected to increase its population significantly and the relaxation dynamics gets slower $[15,16,18,21-$ 32]. A common feature of supercooled liquids is actually the presence of glassy dynamics: the relaxation slows down significantly upon supercooling as a consequence of the emergence of dynamical heterogeneities, since certain portions of the sample relax extremely slowly while others display a much faster dynamics driven by the occurrence of localized clusters of molecules undergoing collectively relaxing motions [17, 33-49]. Supercooled water

\footnotetext{
*appignan@criba.edu.ar
}

has, in fact, been shown to conform to this general scenario, at least in certain regimes [30, 50-53]. As this scenario might suggest (and intuition somehow expects) there should be strong connections between structure and dynamics. But even while the determination of the existence of such a link represents a main question in glass physics and glassy relaxation, it has been extremely difficulty to establish it on firm grounds beyond certain qualitative findings [54-56]. This fact has promoted the proposition of an ingenious indirect approach to first determine which aspect of the dynamic heterogeneity indeed arises from the initial structure [54-56]. This implied the definition of the dynamic propensity [54] as an initial step to then seek for connections with quantities belonging to the system's configuration (static quantities like local structural characteristics or local potential energy) [55, 56]. This indirect measure (calculated by averaging mobility over the so-called isoconfigurational ensemble, a set of many different runs starting from the same initial configuration) represents the dynamic tendency of the water molecules, that is, their probability to perform substantial displacements as determined by the constraints imposed by the system's initial configuration. In other words, the dynamic propensity measures (from its corresponding dynamical tendency) how tightly or loosely a water molecule is bound in a given configuration and has thus provided interesting information on the relationship between structure, dynamics and potential energy surface exploration in model glassformers [46, 47, 54-59]. This approach has also been preliminarily applied to water $[30,52,60]$.

Within the aforementioned context, the availability of reliable structural indicators to quantify the fractions of the two kinds of water "species" and, if possible, to clas- 
sify water molecules (discriminate them as belonging to one of the two mentioned classes) is most desirable and, thus, many different indices have been proposed along the time [21, 22, 28-32, 61-67]. One of such indicators is the local structure index, LSI [21, 22], which has been designed to take advantage of the knowledge that well structured (low local density) molecules present a clear gap between their first two coordination shells, while for unstructured molecules (high local density) such intershell region is populated by the collapse or intrusion of one (or more) second-shell neighbors, thus perturbing the first-shell's tetrahedral coordination. Some time ago, we have shown that when the LSI is applied at the inherent dynamics (local minimizations of potential energy that drive each instantaneous configurations to its inherent structure, IS, the minimum of its corresponding local basin of attraction in the potential energy surface), clear bimodal distributions are obtained [28]. This means that beyond being able to estimate the fraction of the structured and unstructured molecules as most indicators do by proper deconvolution techniques, the presence of a deep minimum between both maxima enables one to safely classify the water molecules in each structural class. This has made the LSI to regain significant popularity, being thenceforth applied at the IS level to many water models with similar positive results (both at the normal liquid and supercooled regimes) as, for example, SPC/E [28, 29], TIP4P - ice [68], TIP5P - Ew [32], TIP4P/2005 [69] and ab initio water model potentials within the framework of density functional theory [70].

On the other hand, the $\zeta$ index $[66,67]$ has been recently introduced, yielding neat bimodal distributions (two maxima separated by a clear minimum) for the TIP5P water model below the melting temperature [67]. A technical advantage of this indicator is that this result is obtained when calculated directly at the real dynamics (at the instant configurations, without need to resort to the IS scheme). For TIP4P $/ 2005$, in turn, the distributions did not yield such a clear bimodality, but nevertheless could be easily decomposed into two gaussian functions [66]. This index has also the advantage, from a conceptual point of view, of explicitly taking into account hydrogen bonding coordination, a major contributor to water structure.

Both the LSI and the $\zeta$ index have been devised to be sensitive to the presence or absence of "interstitial" molecules that might alter the first shell organization. Structured and unstructured states differ thus in the degree of translational order between the first and second shells of the water molecules, a fact that is quantified in different ways by both indicators. Thus, one main goal of the present work is to compare the performance of both structural indicators for different water models, namely SPC/E, TIP4P/2005 and TIP5P. Beyond a direct comparison of their distributions for the different water models (an information that might indicate their relative appropriateness in each case), we shall also relate them to the dynamic propensity [54-56] (as already indicated, a quantity that is not based on structure but that provides information on the local structural/configurational constraints by measuring the probability for motion of the water molecules in a given configuration). Thus, we shall compare the direct structural information provided by the two structural indices with the indirect structural insights reflected by the dynamic propensity values of the molecules in the corresponding configuration. In turn, this leads to the second aim of the present work related to the (longstanding and rather elusive) link between structure and dynamics in glassy relaxation. A few previous applications of the dynamic propensity approach to water have found some mild correlations between this quantity and structural/configurational information [30, 52, 60]. It is interesting to note that in the work of reference [52] the authors found no correlation between the local potential energy of a molecule at the initial configuration and its dynamic propensity (for instance, a molecule that is tightly bound "at the initial configuration" does not necessarily present a low dynamic propensity). The authors thus defined a propensity to be tightly bound "within the isoconfigurational ensemble", which in fact correlated with dynamic propensity: molecules that have a high propensity to be tightly bound are mostly comprised within the least mobile ones [52]. However, while being a nice piece of information, this tendency to be tightly bound represents a quantity averaged over the isoconfigurational ensemble: a function that is not evaluated at the initial time or initial configuration but at later times, when the dynamical heterogeneities are relevant, in several runs starting from such configuration. Moreover, the authors found no significant correlation between the tendency to be loosely bound and dynamic propensity [52] Thus, in the present work we shall relate the propensity measure to the information provided by the structural indicators already mentioned to indeed find the existence of clear correlations between local structure "at the initial configuration" and dynamic propensity. In particular, we shall show that the molecules that display the highest propensity values do correspond with molecules belonging to an unstructured local arrangement, while also finding the existence of evident correlations between well structured molecules and low dynamic propensity.

\section{METHODS}

\section{A. Quantifying the local structure of water molecules: local structure index and $\zeta$ index}

We conducted molecular dynamics (MD) simulations of SPC/E [71], TIP4P/2005 [72] and TIP5P [73] water models by using the GROMACS package version 5.0.2 [74]. Bonds were constrained with the LINCS algorithm and long range electrostatics evaluated with the PME method. The time step used was 2 fs. A modified Berendsen thermostat and a Parrinello-Rahman barostat at 1 bar as reference pressure were used. We built cubic 
boxes of appropriate sizes with periodic boundary conditions and a cutoff of $1 \mathrm{~nm}$ for the short range forces. The SPC/E system consisted of 1050 water molecules while for the TIP4P/2005 TIP4P we employed 1010 molecules and 1087 for TIP5P. These sizes are appropriate for the dynamic propensity approach where a large number of different realization within the isoconfigurational ensemble are demanded (we used 1000 isoconfigurational runs for each temperature). After equilibration for times much larger than the $\alpha$ relaxation time for each temperature, production data runs were produced. In this work we employ two structural indicators based on the degree of translational order up to the second shell. Low density well structured molecules are known to present a clear gap between the two first coordination shells, while in high density molecules this gap gets populated by one or more water molecules from the second shell thus producing a more disordered local structure. The local structure index, LSI [21, 22], has been devised to actually sense the presence or absence of such a gap between the first and second coordination shells. We have applied this index combined with local minimizations of potential energy (by calculating it not at the instantaneous configurations or real dynamics but at the socalled inherent structures, IS, that is, the corresponding local basin of attraction in the potential energy surface for the instantaneous configuration). Under this scheme, the LSI produces clear bimodal distributions of structured and unstructured molecules both for the supercooled and normal liquid regimes [28] for the different water models where it has been applied so far, namely SPC/E [28, 29], TIP4P - ice [68], TIP5P - Ew [32], TIP4P/2005 [69] and ab initio water model potentials within the framework of density functional theory [70]. The LSI index for a central molecule $i$ at time $t, I(i, t)$, is computed by ordering the rest of the molecules depending on the radial distance $r_{j}$ between the oxygen of the molecule $i$ and the oxygen of molecule $j: r_{1}<r_{2}<r_{j}<r_{j+1}<\cdots<r_{n(i, t)}<r_{n(i, t)+1}$, where $n(i, t)$ is chosen so that $r_{n(i, t)}<3.7 \AA<r_{n(i, t)+1}$. Then, $I(i, t)$ is computed as:

$$
L S I(i, t)=I(i, t)=\frac{1}{n(i, t)} \sum_{j=1}^{n(i, t)}[\Delta(j ; i, t)-\bar{\Delta}(i, t)]^{2}
$$

where $\Delta(j ; i, t)=r_{j+1}-r_{j}$ and $\bar{\Delta}(i, t)$ is the average over all molecules of $\Delta(j ; i, t)$. Thus, $I(i, t)$ expresses the inhomogeneity in the radial distribution within the sphere of radius around $3.7 \AA$. A molecule $i$ at time $t$ with well tetrahedral local order and a low local density gives a high value of $I(i, t)$, while a molecule with defective tetrahedral order and high local density yields values of $I(i, t) \sim 0$. As already indicated, differently from Refs. $[21,22]$, we compute the LSI at the inherent structures to avoid the randomizing effect of thermal vibrations, thus effectively removing the fluctuations that prevent a proper identification of the local structure in the real trajectory. Under this scheme, the potential energy surface
(PES) of a many particle system can be partitioned into disjoint basins, where a basin is unambiguously defined as the set of points in configuration space connected to the same local minimum (IS) via a minimization trajectory. Thus, an IS represents the lowest energy configuration of a basin of attraction in the PES, that is, the bottom of the basin. In practical terms, starting from a given instantaneous structure (from the real dynamics or configuration visited by the MD trajectory) we quench this configuration by means of a steepest-descent algorithm until convergence is achieved (until the largest scalar force on any atom is lower than $10 \mathrm{~kJ} / \mathrm{mol} \mathrm{nm}$, with a sufficiently large maximum number of steps of 100000).

In turn, the $\zeta$ index $[66,67]$ is calculated by measuring the difference between the distance $d_{j^{\prime} i}$ of the closest neighbor molecule $j^{\prime}$ not hydrogen-bonded to the central molecule $i$, and the distance $d_{j^{\prime \prime} i}$ of the farthest neighbor molecule $j^{\prime \prime}$ that forms a hydrogen bond (HB) with molecule $i: \zeta(i)=d_{j^{\prime} i}-d_{j^{\prime \prime} i}$, where we shall consider that two water molecules form a $\mathrm{HB}$ when the $\mathrm{O}-\mathrm{O}$ distance is below $3.5 \AA$, and the O-H...O angle is larger than $140^{\circ}$. Thus, this index explicitly incorporates hydrogen bonding. It yielded clear bimodal distributions (two peaks separated by a well defined minimum) for the TIP5P water model within the real dynamics (that is, the instant configurations) below the melting temperature [67]. For TIP4P $/ 2005$, in turn, the distributions did not hold such a clear bimodality, but could be easily decomposed into two gaussian functions [66]. This indicator produces values around $1 \AA$ for low density or structured molecules, but significantly lowers for high density or unstructured molecules, where the first non hydrogen-bonded molecule is placed in the region between the first and the second shell instead of being located at second shell positions (the index even reaches values close to zero or negative, when this molecule intrudes well within the region of the first coordination shell).

\section{B. Dynamic propensity calculations}

The isoconfigurational (IC) method [54] demands performing a series of equal length molecular dynamics trajectories (runs) initiating in the same configuration (identical molecular positions) but with initial molecular momenta randomly chosen from the appropriate Boltzmann distribution. That is, an isoconfigurational (IC) ensemble is built. Then, one calculates the displacements of the molecules at the final time (on the order of the $\alpha$ relaxation time or the timescale of maximum heterogeneity, that is, the time when the non-gaussian parameter is maximum, $\left.t^{*}[42,44]\right)$. If we work at temperatures when the system presents dynamical heterogeneities, mobile and non-mobile molecules will be present in each run but the mobile and non-mobile molecules will differ from run to run since mobility will not be determined by the initial configuration [54]. The dynamic propensity of any given molecule in the initial configuration 
(for a fixed time interval of length $t$ ) is given by [54]: $D P=<\Delta \mathbf{r}_{i}^{2}>_{I C}\left(\right.$ where $<\ldots>_{I C}$ implies an average over the IC and $\Delta \mathbf{r}_{i}^{2}=\left(\mathbf{r}_{i}(t=t)-\mathbf{r}_{i}(t=0)\right)^{2}$ is the squared displacement of molecule $i$ during such time interval). At low temperatures propensities do not distribute in space in a uniform way but high propensity particles tend to arrange in relatively compact regions of the sample [46, 47, 54-59]. Thus, even when molecular mobility is not reproducible from run to run, the spatial variation of propensity is indeed completely determined by the initial configuration. This fact speaks of the existence of a clear influence of structure on dynamics [46, 47, 54-59]. We have shown [46, 47] that the fact that the spatial distribution of propensity is determined by the initial configuration (for time intervals on the order of the $\alpha$ relaxation) derives from the local exploration performed by the system of its potential energy surface (metabasin confinement). That is, all the diverging trajectories from a common origin of the IC are nonetheless confined to the same metabasin for most of the time under study. In the present work we decided to calculate propensity values by using 1000 IC runs for each temperature and each water model. The total times for the runs corresponded to the timescale when the water molecules have moved on average one intermolecular distance for each case (this timescale, consistent with the mean cage escape time, would be close to the $\alpha$ relaxation time at each studied temperature $[46,47,51])$. Specifically, these times were: $600 \mathrm{ps}$ for $\mathrm{SPC} / \mathrm{E}$ at $\mathrm{T}=210$ $\mathrm{K} ; 35 \mathrm{ps}$ for $\mathrm{SPC} / \mathrm{E}$ at $\mathrm{T}=240 \mathrm{~K}$; $50 \mathrm{ps}$ for $\mathrm{TIP} 4 \mathrm{P} / 2005$ at $\mathrm{T}=247 \mathrm{~K} ; 10 \mathrm{ps}$ for $\mathrm{TIP} 4 \mathrm{P} / 2005$ at $\mathrm{T}=281 \mathrm{~K} ; 20 \mathrm{ps}$ for TIP5P at $\mathrm{T}=267 \mathrm{~K} ; 5 \mathrm{ps}$ for TIP5P at $\mathrm{T}=305 \mathrm{~K}$. In all cases, to correlate dynamic propensity results with the structural information provided by the LSI and the $\zeta$ index we calculated such indices at the initial configuration of the corresponding IC (the configuration from which the 1000 runs started and whose dynamic constraints are being reflected by the results of the dynamic propensity method).

\section{RESULTS}

Fig. 1 compares the distributions of the LSI index and the $\zeta$ index for the three water models for a series of temperatures from above to below the corresponding melting temperatures (the melting temperatures for TIP5P, TIP4P/2005 and SPC/E have been estimated to be respectively $T_{m}=272 \mathrm{~K}, T_{m}=250.5 \mathrm{~K}$ and $T_{m}=214 \mathrm{~K}$ [75]). The LSI index is calculated at the inherent dynamics ( IS, where it provides a clear bimodal behavior improving significantly the results as compared to the real dynamics case) while the $\zeta$ index is computed directly at the instantaneous configurations or real dynamics as defined (employment of the IS scheme does not improve the results as in the case of the LSI ). In all cases it is evident that both structural indicators display signs of bimodality, being suitable for the estimation of the frac-
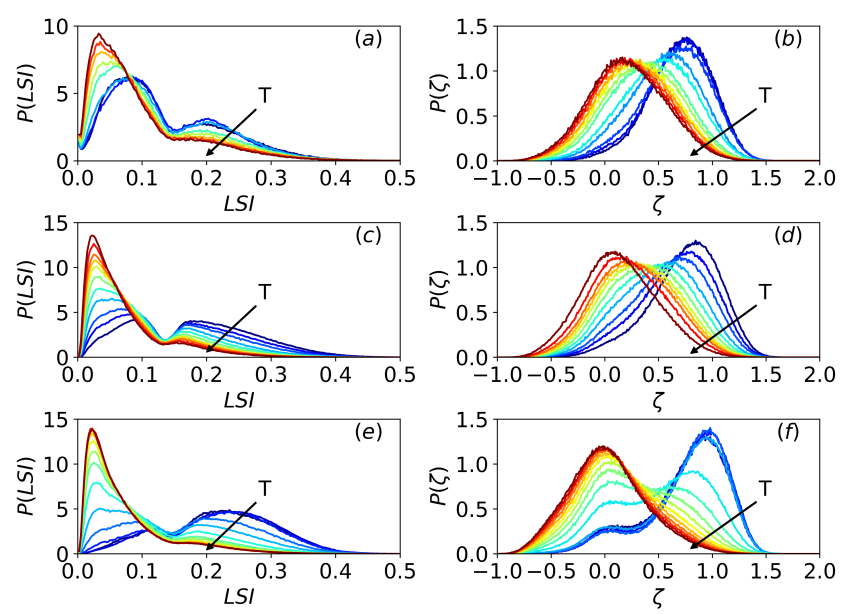

FIG. 1: LSI (calculated at the inherent dynamics) and $\zeta$ index for SPC/E, TIP4P/2005 and TIP5P for a series of temperatures from below to above the corresponding melting points. The arrows depict the direction of increasing temperature. The temperature ranges are as follows: a) SPC/E and LSI: T from 180 to $250 \mathrm{~K}$; b)

SPC/E and $\zeta$ index: from 180 to $300 \mathrm{~K}$; c) TIP4P/2005 and LSI: 210 to $350 \mathrm{~K}$; d) TIP4P/2005 and $\zeta$ index: 210 to $350 \mathrm{~K}$; e) TIP5P and LSI: 200 to $330 \mathrm{~K}$; ) TIP5P and $\zeta$ index: 200 to $340 \mathrm{~K}$. The LSI is given in units of $\AA^{2}$, while the $\zeta$ index is given in $\AA$.

tion of high and low density water molecules after proper deconvolution. The LSI, additionally, displays a neat bimodality for all water models, with a deep minimum (isosbestic point) between the two maxima corresponding to structured and unstructured molecules. This fact enables one to employ this index to classify molecules in structured and unstructured by considering as a threshold the LSI value of the minimum (if no minimum would be present, the distributions of the two kinds of molecules would overlap significantly, thus precluding a proper classification). The $\zeta$ indicator also displays a nice bimodality for the TIP5P model at temperatures roughly below the melting point (while for higher $\mathrm{T}$ this behavior is progressively lost). However, the performance of this indicator is quite different for both SPC/E and TIP4P/2005 water models, where no clear bimodality is present in the corresponding distributions. Thus, while being able to estimate the population of the two kinds of molecules, it lacks the evident classification capability displayed by the LSI.

Given the differences in the distributions of the two indicators for the different water models, as evident from Fig. 1, it is interesting to further compare their correlations with another kind of structural indicator. However, by definition (provided the particular structural information involved in its construction), other structurallybased indicators might already imply a different degree of similarity to each of the two indices. A most interesting possibility to overcome this limitation is given by the 

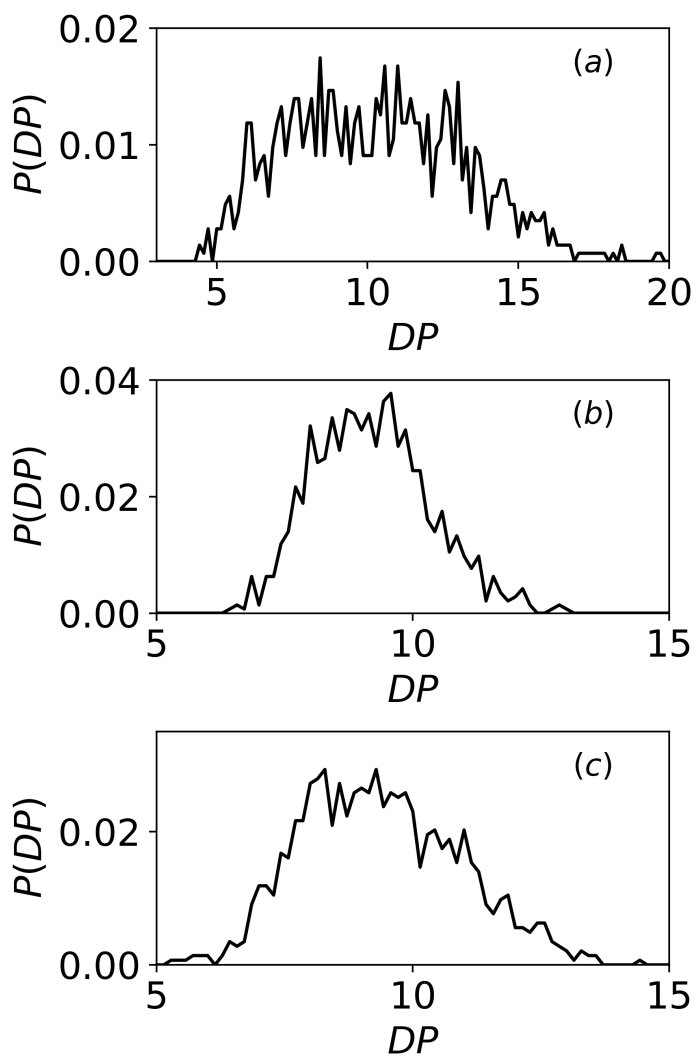

FIG. 2: Typical dynamic propensity distributions for: (a) $\mathrm{SPC} / \mathrm{E}(\mathrm{T}=210 \mathrm{~K})$; (b) TIP4P $/ 2005(\mathrm{~T}=246 \mathrm{~K})$ and (c) TIP5P $(\mathrm{T}=267 \mathrm{~K})$. Dynamic propensity is given in units of $\AA^{2}$.

dynamic propensity measure, $D P$. This quantity has the advantage that it provides indirect structural information since it is not built upon any structural parameter but reveals the structural constraints present in the system from the corresponding dynamical behavior. In other words, the dynamic propensity measures how tightly or loosely held is each molecule in a particular configuration by computing its tendency to move away from its position in such initial configuration. Thus, we shall study correlations between the dynamic propensity values of the molecules as calculated for a given initial configuration (the initial configuration of all the isocofigurational runs and whose dynamic constraints are being reflected by the IC method) and the corresponding LSI and $\zeta$ values, computed at such same initial configuration. Beyond the virtue of providing a means to compare the performance of the two structural indicators in an unbiased way, this procedure would also enable us to seek for connections between structure and dynamics at low temperatures, an issue of major concern in the context of supercooled liquids and glass physics.

In Fig. 2 we show typical results for the dynamic propensity distributions at low temperatures. The distributions of dynamic propensity are known to be sharp and gaussian-like at high temperatures while they get progressively non-gaussian, with a tail to large propensity values, as temperature is lowered in the supercooled regime [56]. Fig. 2 corresponds to propensity distributions for the three water models under study for temperatures slightly below their corresponding melting points. Fig. 3, in turn, provides the correlations of the two structural indicators (LSI and $\zeta$ ) with dynamic propensity for the three water models (in each case we consider a temperature above the corresponding melting point and also a temperature lower than the melting point).

In which concerns the LSI indicator, our results confirm the existence of correlations with dynamic propensity for all three water models: High LSI molecules (structured) tend to display low propensity values (low tendency for motion) while low LSI molecules markedly exhibit high propensity ones. In particular, the bimodality of the index is evident from the two spots present in the plots (the high LSI molecules belong to the smaller spot located preferentially at low propensity values, while the spot for the low LSI molecules is larger and presents an elongated or oblong shape that deforms following a negative slope). Notably, the reluctance of high propensity molecules to display high LSI values characteristic of well-structured molecules is particularly evident. In the case of the $\zeta$ index, in turn, we find a similar behavior for TIP5P (particularly at the lowest temperature, below the melting point) with insinuation of the two spots characteristic of clearly bimodal behavior. Concerning TIP4P $/ 2205$, we observe a single elongated spot following a negative slope, thus also implying certain correlation between high LSI and low propensity values (and also between low LSI and high propensity).

The global correlations between dynamic propensity and structural information would be quite small if we include all the particles for the plots of Fig. 3. This is so since only the molecules with extreme behavior display good correlations (the lowest propensity molecules and, particularly, the highest propensity ones). Thus, to further reveal the existence of a link between structural information and dynamic propensity, we focus on the molecules with propensity values within the highest and lowest $10 \%$. In Fig. 4 we plot the LSI and $\zeta$ distributions for the molecules with dynamic propensity values within the highest $10 \%$ (red curves) and also for the $10 \%$ lowest propensity molecules (blue curves) for the three water molecules at chosen temperatures. For space reasons, for each water model we only display results for one of the temperatures employed in Fig. 3, but the behavior does not change significantly for the other case. For comparison, we also include the corresponding distributions of the dynamic propensity for all the water molecules (black curves). Regarding the LSI index, it is immediately evident that the molecules with the highest propensity values (10\% highest propensity molecules) exhibit a basically unimodal LSI distribution, that is, they belong almost exclusively to the left peak of the LSI distribution, the one that encompasses the unstruc- 

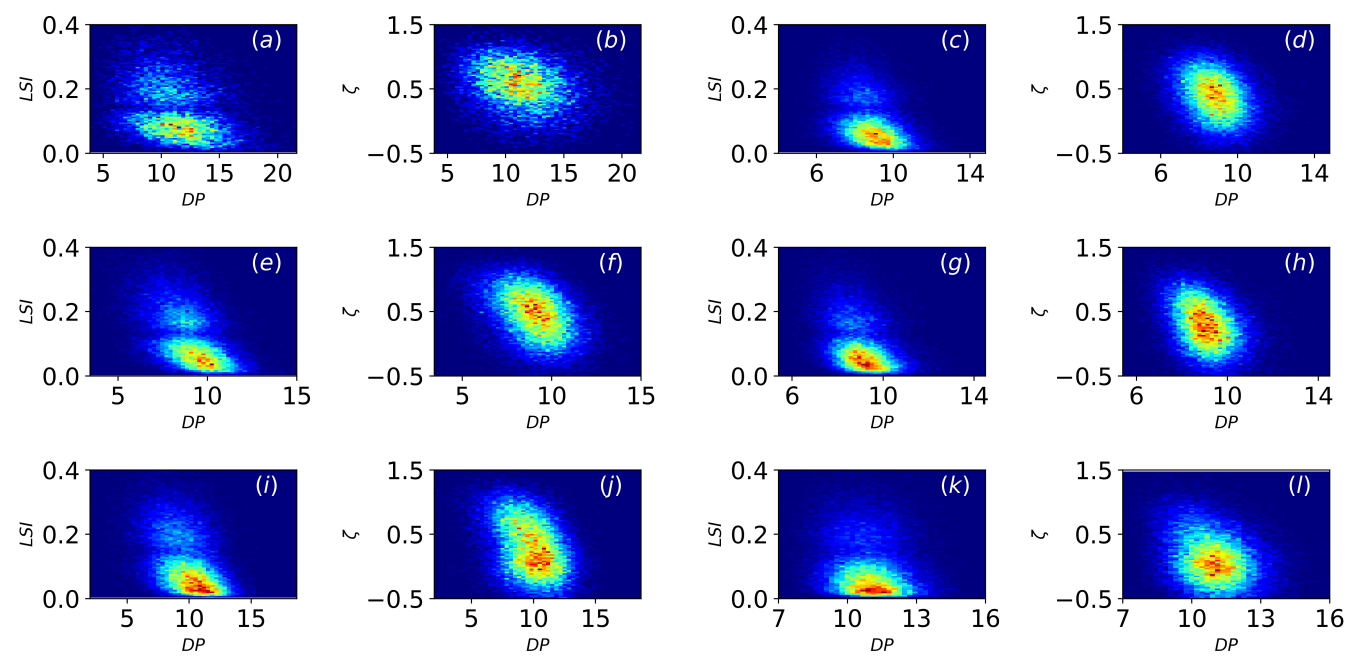

FIG. 3: Correlation maps between LSI and the dynamic propensity (DP) and between the $\zeta$ index and DP. Each plot row shows results for a different water model, including one temperature that is above and another below the corresponding melting point. (a) SPC/E: LSI vs DP for T=210 K; (b) SPC/E: $\zeta$ vs DP for T=210 K; (c) SPC/E:

LSI vs DP for T=240 K; (d) SPC/E: $\zeta$ vs DP for T=240 K; (e) TIP4P/2005: LSI vs DP for T=246 K; (f)

TIP4P/2005: $\zeta$ vs DP for T=246 K; (g) TIP4P/2005: LSI vs DP for T=281 K; (h) TIP4P/2005: $\zeta$ vs DP for $\mathrm{T}=281 \mathrm{~K}$; (i) TIP5P: LSI vs DP for $\mathrm{T}=267 \mathrm{~K}$; (j) TIP5P: $\zeta$ vs DP for $\mathrm{T}=267 \mathrm{~K}$; (k) TIP5P: LSI vs DP for $\mathrm{T}=305$ $\mathrm{K}$; (l) TIP5P: $\zeta$ vs DP for T=305 K.

tured molecules. The molecules with the less propensity values, in turn, still display a bimodal LSI distribution but the peak for the structured molecules (right peak of the LSI distribution) is significantly increased at the expense of the other as compared to the LSI distribution for all the water molecules. These results are similar for all three water models. Regarding the $\zeta$ index, in turn, we learn the existence of a similar behavior for TIP5P water model, with a nearly unimodal unstructured-like distribution for the high propensity molecules and a bimodal distribution for the low propensity molecules, the latter being clearly enriched in the structured class as compared to the full $\zeta$ distribution for all the molecules. For the other two water models, however, the $\zeta$ distributions for the high and low propensity molecules show peaks to the left and to the right of the peak for the $\zeta$ distribution of the full set of water molecules, respectively. While this split does not lead these peaks completely to typical unstructured and structured molecules ( $\zeta$ values around zero and one, respectively, as for the TIP5P case), it still conforms to the scenario of a connection between local structure and dynamic propensity (mainly unstructured high propensity molecules and mainly well structured low propensity ones). It is worth noting that the full $\zeta$ distributions present no clear bimodality for such cases.

Finally, in Fig. 5 we present an example of a threedimensional configuration for $\mathrm{SPC} / \mathrm{E}$ water at $\mathrm{T}=240 \mathrm{~K}$ where we plot the spatial distribution of the molecules within the $10 \%$ highest propensity (coloured in red) and $10 \%$ lowest propensity (coloured in blue if they belong to the structured peak of the LSI, that is, the right peak of the LSI distribution of Fig. 4 (a), or light blue if their LSI value is located within the unstructured peak, the left-side peak of Fig. 4 (a)). From a qualitative point of view, evident clusters of like molecules can be observed. Taken together, blue and light blue molecules exhibit certain tendency to arrange in more compact clusters (also with the presence of some string-like regions) while the red ones seem to arrange themselves in more string-like clusters similar to that found for mobile molecules in glassy systems [42], or branched string-like ones as already observed in water [76]. We stress that here we are plotting propensity values (averaged behavior within the iso-configurational ensemble, that is, over many runs starting from a single initial configuration) and not mobility values on a single run as in such cases. We have also found a similar situation for the other two water models.

\section{CONCLUSIONS}

The present study has compared the behavior of two structural indicators built upon measures of the translational order up to the second coordination shell of the water molecules. We have found that the LSI index, when combined with energy minimizations, provides clear bimodal distributions with two maxima separated by a deep minimum in all three water models studied (SPC/E, TIP4P/2005 and TIP5P). This enables one not only to estimate the fraction of structured (low local density) and unstructured (high local density) water molecules by proper deconvolution, but to also classify the molecules 

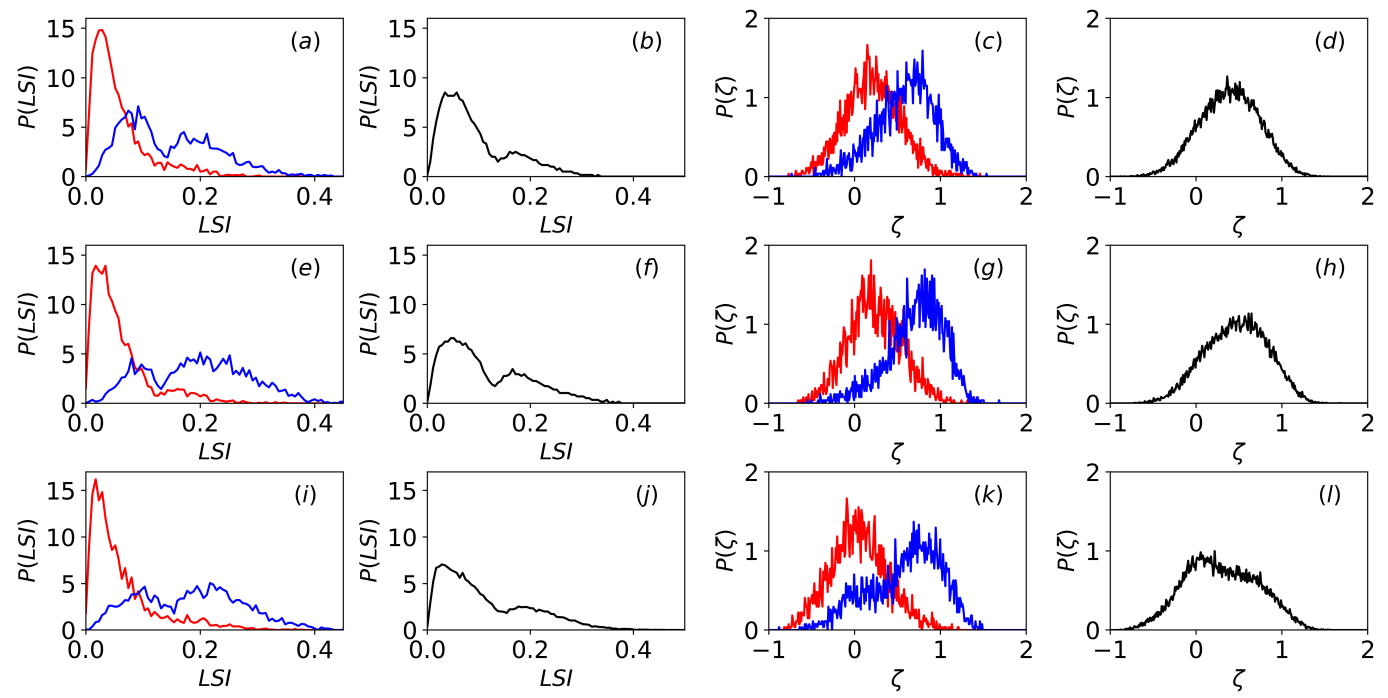

FIG. 4: LSI and $\zeta$ index distributions for the molecules within the $10 \%$ highest propensity values (red), for the molecules within the $10 \%$ lowest propensity values (blue) and for all the water molecules (black). Each row corresponds to a different water model. (a) and (b): LSI distributions for SPC/E at T=240 K; (c) and (d): $\zeta$ distributions for SPC $/ \mathrm{E}$ at T=240 K; (e) and (f): LSI distributions for TIP4P $/ 2005$ at $\mathrm{T}=246 \mathrm{~K}$; (g) and (h): $\zeta$ distributions for TIP4P/2005 at T=246 K; (i) and (j): LSI distributions for TIP5P at T=267 K; (k) and (l): $\zeta$ distributions for TIP5P at $\mathrm{T}=267 \mathrm{~K}$.

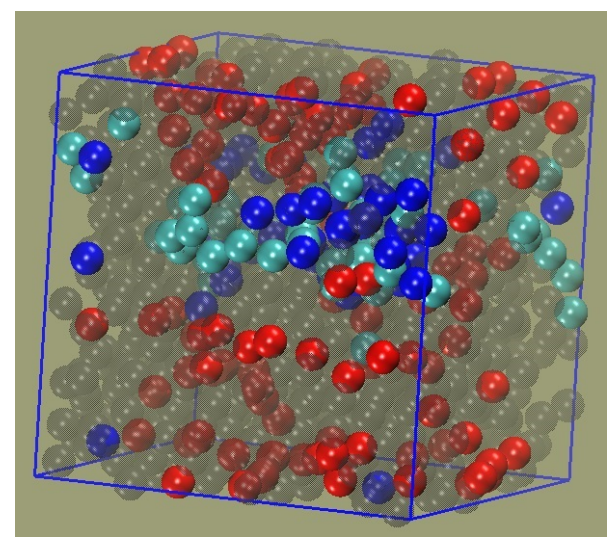

FIG. 5: 3D plot of a typical configuration with the $10 \%$ highest propensity molecules colored in red, and the 10 $\%$ lowest propensity molecules colored in blue (if they belong to the right peak of the LSI distribution or structured molecules) or light blue (if they are comprised within the left peak corresponding to the unstructured molecules). The red molecules are almost all within the left peak of the LSI distribution. We also indicate the position of the rest of the water molecules (in a pale gray color). The model is $\mathrm{SPC} / \mathrm{E}$ at $\mathrm{T}=240$ K.

within the two classes indicated. The $\zeta$ index (which introduces a conceptual advance by incorporating hydrogen bonding in its definition), in turn, yields a similar performance for the TIP5P water model but gives less neat bimodal distributions for the other two models.
Thus, while indeed enabling the estimation of the two fractions of molecular classes by proper deconvolution in $\mathrm{SPC} / \mathrm{E}$ and TIP4P/2005, this fact prevents from performing a direct molecular classification. We have also compared the results for the two indicators with a quantity that is based not on direct structural information but that represents a dynamical manifestation of the underlying structural constraints: the dynamic propensity. This measure provides the tendency (probability) for motion of the water molecules in a given configuration, thus indicating how loosely or tightly engaged (how constrained) is each molecule at such initial configuration. We have shown that both indices display evident correlations with dynamic propensity, as structured molecules display a preference for low dynamic propensity values and, more markedly, unstructured molecules exhibit a very prominent tendency to present a high dynamic propensity. These results are more explicitly evident for the cases when the indices display a neat bimodality (LSI for all models and $\zeta$ index for TIP5P, particularly at low temperature). The existence of neat correlations between structural information and dynamic propensity is particularly relevant for the supercooled regime where the finding of a link between structure and dynamics (with dynamics that vary in orders of magnitude with the supercooling) has long remained as a tough undertaking.

\section{ACKNOWLEDGMENTS}

The authors acknowledge support form CONICET, UNS and ANPCyT (PICT2015/1893 and 
PICT2017/3127).

[1] P. Ball, Proc. Natl. Acad. Sci. U.S.A. 11413327 (2017).

[2] D. M. Huang, D. Chandler, Proc. Natl. Acad. Sci. U.S.A. 978324 (2000).

[3] X. Huang, C. J. Margulis and B. J. Berne, Proc. Natl. Acad. Sci. U.S.A. 10011953 (2003).

[4] N. Giovambattista, P. G. Debenedetti, C. F. Lopez, and P. J. Rossky, Proc. Natl. Acad. Sci. U.S.A 1052274 (2008).

[5] A. Bizzarri and S. Cannistraro, J. Phys. Chem. B 106 6617 (2002).

[6] D. Vitkup, D. Ringe, G. A. Petsko and M. Karplus, Nat. Struct. Biol. 734 (2000).

[7] N. Choudhury and B. Montgomery Pettitt, J. Phys. Chem. B 1096422 (2005).

[8] H. E. Stanley, P. Kumar, L. Xu, Z. Yan, M. G. Mazza, S. V. Buldyrev, S. -H. Chen and F. Mallamace, Physica A 386729 (2007).

[9] E.P. Schulz, L.M. Alarcón, G.A. Appignanesi, Eur. Phys. J. E 34, 114 (2011).

[10] D.C. Malaspina, E.P. Schulz, L.M. Alarcón, M.A. Frechero, G.A. Appignanesi, Eur. Phys. J. E 32, 35 (2010).

[11] L.M. Alarcón, D.C. Malaspina, E.P. Schulz, M.A. Frechero, G.A. Appignanesi, Chem. Phys. 388, 47 (2011).

[12] S.R. Accordino, D.C. Malaspina, J.A. Rodriguez Fris, L.M. Alarcón, and G. A. Appignanesi, Phys. Rev. E 85, 031503 (2012).

[13] S.R. Accordino, J.M. Montes de Oca, J.A. Rodriguez Fris, G.A. Appignanesi, J. Chem. Phys. 143, 154704 (2015).

[14] J.M. Montes de Oca, C.A. Menéndez, S.R. Accordino, D.C. Malaspina and G.A. Appignanesi, Eur. Phys. J. E 40, 78 (2017).

[15] P. H. Poole, F. Sciortino, U. Essmann and H. E. Stanley, Nature 360, 324 (1992)

[16] P. G. Debenedetti and H. E. Stanley, Physics Today 56, 40 (2003)

[17] P. G. Debenedetti, Metastable Liquids, Princeton University Press, Priceton, NJ (1996).

[18] O. Mishima and H. E. Stanley, Nature 396329 (1998).

[19] C. A. Angell, Chem. Rev. 1022627 (2002).

[20] C. A. Angell, Annu Rev. Phys. Chem. 55559 (2004).

[21] E. Shiratani and M. Sasai, J. Chem. Phys. 1047671 (1996).

[22] E. Shiratani and M. Sasai, J. Chem. Phys. 108, 3264 (1998).

[23] M. Sasai, Physica A 285315 (2000).

[24] M. Sasai, J. Chem. Phys. 11810651 (2003).

[25] H. Tanaka, Phys. Rev. Lett. 805750 (1998).

[26] H. Tanaka, Europhys. Lett. 50340 (2000).

[27] H. Tanaka, J. Chem. Phys. 112799 (2000).

[28] G. A. Appignanesi, J. A. Rodriguez Fris and F. Sciortino, Eur. Phys. J. E 29305 (2009).

[29] S. R. Accordino, J. A. Rodriguez Fris, F. Sciortino and G. A. Appignanesi, Eur. Phys. J. E 3448 (2011)

[30] D.C. Malaspina, J.A. Rodriguez Fris, G. A. Appignanesi and F. Sciortino, EuroPhys. Lett. 88, 16003 (2009).
[31] S.R. Accordino, D.C. Malaspina, J.A. Rodriguez Fris and G. A. Appignanesi, Phys. Rev. Lett. 106, 029801 (2011).

[32] J.M. Montes de Oca, J.A. Rodriguez Fris, S.R. Accordino, D.C. Malaspina and G.A. Appignanesi, Eur. Phys. J. E 39, 124 (2016).

[33] C. A. Angell, Science 267, 1924 (1995).

[34] C. A. Angell, K. L. Ngai, G. B. McKenna, P. F. McMillan, and S. W. Martin, J. App. Phys. 88, 3113 (2000).

[35] E. R. Weeks, J. C. Crocker, A. C. Levitt, A. Schofield, and D. A. Weitz, Science 287, 627 (2000).

[36] H. Sillescu, J. Non-Cryst. Solids 243, 81 (1999).

[37] M. D. Ediger, Ann. Rev. Phys. Chem. 51, 99 (2000).

[38] S. C. Glotzer, Physics of Non-Crystalline Solids 9, J. Non-Cryst. Solids 274, 342 (2000).

[39] E. Hempel, G. Hempel, A. Hensel, C. Schick, and E. Donth, J. Phys. Chem. B 104, 2460 (2000).

[40] R. Richert, J. Phys.: Condens. Matter 14, R703 (2002).

[41] W. Kob, C. Donati, S. J. Plimpton, P. H. Poole, and S. C. Glotzer, Phys. Rev. Lett. 79, 2827 (1997).

[42] C. Donati, J. F. Douglas, W. Kob, S. J. Plimpton, P. H. Poole, and S. C. Glotzer, Phys. Rev. Lett. 80, 2338 (1998).

[43] C. Donati, S. C. Glotzer, and P. H. Poole, Phys. Rev. Lett. 82, 5064 (1999).

[44] G. A. Appignanesi, J. A. Rodriguez Fris, R. A. Montani, and W. Kob, Phys. Rev. Lett. 96, 057801 (2006).

[45] G. A. Appignanesi and J. A. Rodriguez Fris, J. Phys.: Condens. Matter 21, 203103 (2009).

[46] G.A. Appignanesi,J.A Rodriguez Fris and M.A. Frechero, Phys. Rev. Lett. 96, 237803 (2006).

[47] J.A. Rodriguez Fris, L.M. Alarcón and G.A. Appignanes, Phys. Rev. E 76, 011502 (2007).

[48] J.A. Rodriguez Fris, G.A. Appignanesi and E.R. Weeks, Phys. Rev. Lett. 107, 065704 (2011).

[49] J.A Rodriguez Fris, E.R. Weeks, F. Sciortino and G.A. Appignanesi, Phys. Rev. E 97, 060601(R) (2018).

[50] E. La Nave and F. Sciortino, J. Phys. Chem. B 108, 19663 (2004).

[51] J. A. Rodriguez Fris, G. A. Appignanesi, E. La Nave and F. Sciortino, Phys. Rev. E 75041501 (2007).

[52] G. S. Matharoo, M.S. Gulam Razul, and P. H. Poole, Phys. Rev. E 74, 050502(R) (2006).

[53] J. M. Montes de Oca, S. R. Accordino, G. A. Appignanesi, P. H. Handle, and F. Sciortino, J. Chem. Phys. 150, 144505 (2019).

[54] A. Widmer-Cooper, P. Harrowell and H. Fynewever, Phys. Rev. Lett. 93, 135701 (2004).

[55] A. Widmer-Cooper and P. Harrowell, Phys. Rev. Lett. 96, 185701 (2006).

[56] A. Widmer-Cooper and P. Harrowell, J. Chem. Phys. 126, 154503 (2007).

[57] M. A. Frechero, L. M. Alarcón, E. P. Schulz and G. A. Appignanesi, Physical Review E 75, 011502 (2007).

[58] J. A. Rodriguez Firs, L. M. Alarcón and G. A. Appignanesi. J. Chem. Phys. 130024108 (2009).

[59] D. Malaspina, E. P. Schulz, M. A. Frechero and G. A. Appignanesi. Physica A 3883325 (2009). 
[60] M. Fitzner, G.C. Sosso, S.J. Cox and A. Michaelides, Proc. Natl. Acad. Sci USA 116, 2009 (2019)

[61] P.-L. Chau and A. J. Hardwick, Mol. Phys. 93511 (1998).

[62] J. R. Errington and P. G. Debenedetti, Nature 409318 (2001).

[63] I. Naberukhin Yu, V. P. Voloshin and N. N. Medvedev, Mol. Phys. 73917 (1991).

[64] A. Oleinikova and I. Brovchenko, J. Phys.: Condensed Matter 18 S2247 (2006).

[65] M. J. Cuthbertson and P. H. Poole, Phys. Rev. Lett. 106, 115706 (2011)

[66] J. Russo and H. Tanaka, Nat. Comm. 5, 3556 (2014).

[67] R. Shi and H. Tanaka, J. Chem. Phys. 148, 124503 (2018).

[68] J. Gelman Constantin, A. Rodriguez Fris, G. Appignanesi, M. Carignano, I. Szleifer and H. Corti, Eur. Phys.
J. E 34126 (2011).

[69] K. T. Wikfeldt, A. Nilsson and L. G. M. Pettersson, Phys. Chem. Chem. Phys. 1319918 (2011).

[70] B. Santraa, R. A. DiStasio Jr., F. Martellia and R. Car Mol. Phys. 1132829 (2015).

[71] H. J. C. Berendsen, J. R. Grigera and T. P. Straatsma, J. Phys. Chem. 91, 6269 (1987).

[72] J. L. F. Abascal and C. Vega, J. Chem. Phys. 123 (2005) 234505

[73] M. W. Mahoney and W. L. Jorgensen, J. Chem. Phys. 112, 8910 (2000).

[74] H. J. C. Berendsen, D. van der Spoel and R.van Drunen, Comp. Phys. Comm. 91 43, 1995.

[75] R. G. Fernandez, J. L. F. Abascal and C. Vega, J. Chem. Phys. 124144506 (2006).

[76] N. Giovambattista, S. V. Buldyrev, F. W. Starr and H. Eugene Stanley, Phys. Rev. Lett. 90, 085506 (2003). 